\title{
EFEITOS DE SOLOS E TOPOGRAFIA SOBRE A DISTRIBUIÇÃO DE ESPÉCIES ARBÓREAS EM UM FRAGMENTO DE FLORESTA ESTACIONAL SEMIDECIDUAL, EM LUMINÁRIAS, MG'
}

Luciene Alves Rodrigues², Douglas Antônio de Carvalho ${ }^{3}$, Ary Teixeira de Oliveira Filho e Nilton Curi $^{5}$

\begin{abstract}
RESUMO - O objetivo deste trabalho foi analisar correlações da distribuição de espécies arbóreas com variáveis ambientais em um fragmento de floresta estacional semidecidual conhecido como "Mata do Galego", o qual possui cerca de 77 ha e está localizado a $21^{\circ} 29^{\prime} \mathrm{S}$ e $44^{\circ} 55^{\prime} \mathrm{W}$, às margens do rio Ingaí. O levantamento da comunidade arbórea, dos solos e da topografia foi realizado por meio de amostragem sistemática, em 32 parcelas de 20 x 20 m, distribuídas em três transeções, da margem do rio até a borda da mata. Foram registrados todos os indivíduos arbóreos, vivos, com circunferência à altura do peito $(\mathrm{CAP}) \geq 15,5 \mathrm{~cm}$. As variáveis químicas e físicas do solo foram obtidas das análises de amostras superficiais de solo $(0-20 \mathrm{~cm})$ de cada parcela. As variáveis topográficas foram obtidas por meio de um levantamento topográfico dentro e entre parcelas. Foram inventariados 2.343 indivíduos de 159 espécies, 50 famílias e 109 gêneros. As análises de gradientes, realizadas por meio de análise de componentes principais, análise de correspondência canônica e análise de correspondência retificada, indicaram que as parcelas localizadas em áreas de transição com campo de altitude foram as que mais se diferenciaram das demais. As variáveis relacionadas com a acidez e textura do solo apresentaram maior correlação com a distribuição das espécies.
\end{abstract}

Palavras-chave: Floresta semidecidual, comunidade arbórea e variáveis ambientais.

\section{EFFECTS OF SOILS AND TOPOGRAPHY ON THE DISTRIBUTION OF TREE SPECIES IN A FRAGMENT OF TROPICAL SEASONAL FOREST IN LUMINÁRIAS, SE BRAZIL}

\begin{abstract}
The objective of the present study was to analyze the correlations between tree species distribution and environmental variables in a fragment of tropical seasonal forest in Luminárias, SE Brazil. The fragment, locally known as "Mata do Galego", has an area of c. 77ha, and is located on the margin of the Ingai River at the coordinates $21^{\circ} 29^{\prime} \mathrm{S}$ and $44^{\circ} 55^{\prime} W G$. The survey of the tree community, soils and topography was carried out through systematic sampling with 32 sample plots with $20 \times 20$ m distributed on three transects which extended from the river margin to the forest edge. In these plots, all living individual trees with circumference at breast height $(\mathrm{cbh}) \geq 15.5 \mathrm{~cm}$ were recorded. Soil chemical and physical variables were obtained from the analysis of topsoil samples $(0-20 \mathrm{~cm})$ taken from the center of each plot. Topographic variables were obtained from a topographic survey of the plots and adjacent areas. The survey recorded 2343 individuals
\end{abstract}

\footnotetext{
${ }^{1}$ Recebido em 22.11.2005 e aceito para publicação em 13.09.2006.

${ }^{2}$ Instituto Brasileiro do Meio Ambiente e dos Recursos Naturais Renováveis - IBAMA, 78600-000 Barra do Garças-MT. E-mail: <luarmg@uol.com.br>.

${ }_{3}^{3}$ Departamento de Biologia da Universidade Federal de Lavras (UFLA), 37200-000 Lavras-MG. E-mail: <douglasc@ ufla.br>.

${ }^{4}$ Departamento de Ciências Florestais da UFLA, 37200-000 Lavras-MG. E-mail: <ary@ufla.br>.

${ }^{5}$ Departamento de Ciências dos Solos da UFLA, 37200-000 Lavras-MG. E-mail: <niltcuri@ufla.br>.
} 
belonging to 159 species, 109 genera, and 50 families. The gradient analysis; performed through principal components analysis (PCA), canonical correspondence analysis (CCA), and detrended correspondence analysis (DCA); indicated that the plots located near the forest edge and transitional to the montane grasslands are the most differentiated. Variables related to soil $\mathrm{pH}$ and texture produced the strongest correlations with the distribution of tree species.

Keywords: Tropical seasonal forest, tree species and environmental variables.

\section{INTRODUÇÃO}

Vários estudos têm apontado que um dos principais fatores atuando na composição florística e estrutura das florestas é a heterogeneidade ambiental, cujos efeitos podem ser observados mesmo no interior de pequenos fragmentos (OLIVEIRA FILHO et al., 1994b; 1998; DURIGAN et al., 2000; BOTRELet al., 2002; CARVALHO et al., 2005; ROCHA et al., 2005). Essa heterogeneidade é resultado da diversidade de fatores que interagem nas comunidades e a resposta das espécies a esses fatores faz que cada local tenha características próprias e características que são comuns a outros locais, possibilitando observar tendências. Essas tendências podem responder a algumas perguntas e gerar várias outras, funcionando com força motriz para novos estudos.

Numa escala local, a topografia tem sido considerada como a mais importante variável na distribuição espacial e na estrutura das florestas tropicais, porque ela comumente corresponde às mudanças nas propriedades dos solos, particularmente no regime de água e na fertilidade. A correlação entre distribuição de espécies arbóreas e variáveis de solos e de topografia tem sido sucessivamente demonstrada em numerosos estudos de florestas tropicais (CLARK et al., 1998; Van Den BERG e OLIVEIRA FILHO, 1999; OLIVEIRA FILHO et al., 2001; ESPÍRITO SANTO et al., 2002; SOUZA et al., 2003; CARVALHO et al., 2005).

Na região do Alto Rio Grande, Sul de Minas Gerais, diversos trabalhos já foram desenvolvidos em fragmentos florestais, com o propósito de se conhecer a ecologia das espécies arbóreas nativas, visando à seleção de espécies a serem utilizadas em posteriores trabalhos de revegetação (OLIVEIRA FILHO et al., 1994a,b; 1998; 2001). Esses estudos consistem, basicamente, na determinação de "habitats" preferenciais, para crescimento e estabelecimento das espécies, identificando correlações entre categorias de solos, propriedades dos solos, topografia (drenagem) e estrutura da comunidade arbórea.

R. Árvore, Viçosa-MG, v.31, n.1, p.25-35, 2007
O presente estudo foi conduzido em um fragmento florestal adjacente ao curso do rio Ingaí, no Município de Luminárias, MG, com o objetivo de verificar as correlações entre as variações da estrutura da comunidade arbórea (CAP $\geq 15,5 \mathrm{~cm}$ ) e as variáveis ambientais relacionadas ao substrato. Compreende-se como substrato o conjunto de variáveis topográficas e pedológicas determinantes do "status" nutricional do solo e de seu regime hídrico. Resultados sobre a florística e a estrutura do fragmento são apresentados em Rodrigues et al. (2003).

\section{MATERIAL E MÉTODOS}

\subsection{Local de estudo}

A área de estudo localiza-se nas Fazendas Barreiro e Morro Grande, Município de Luminárias, Minas Gerais, às margens do rio Ingaí, um dos afluentes do rio Grande, nas coordenadas $21^{\circ} 29^{\prime} \mathrm{S}$ e $44^{\circ} 55^{\prime} \mathrm{W}$, entre 880 e 1.001 m de altitude (Figura 1A). O fragmento florestal é conhecido localmente por "Mata do Galego" e possui aproximadamente 77 ha. O desnível entre a borda do fragmento, no alto da encosta, até o rio é de $121 \mathrm{~m}$, o que origina um gradiente ambiental e vegetacional, que inclui representações da Floresta Estacional Semidecidual Aluvial e Floresta Estacional Semidecidual Montana.

Segundo informações locais, houve a retirada de madeira na Mata do Galego. O desmatamento teve início no final da década de 1930, e nas áreas abertas plantavamse milho, arroz e feijão, por mais ou menos dois anos. Após esse período, a área era abandonada e nova área era desmatada e cultivada. Esses ciclos duravam aproximadamente 10 anos. Foi aberta uma estrada dentro da floresta, margeando o rio Ingaí, por onde se retirava madeira e se transportavam seixos extraídos do rio. Atualmente, não ocorre exploração de seixos nem de madeira, mas há entrada de gado de uma das fazendas. Uma das extremidades da floresta tem ligação com remanescentes menores, uma das bordas tem interface com campo de altitude e outra, com áreas agrícolas. 
(A)
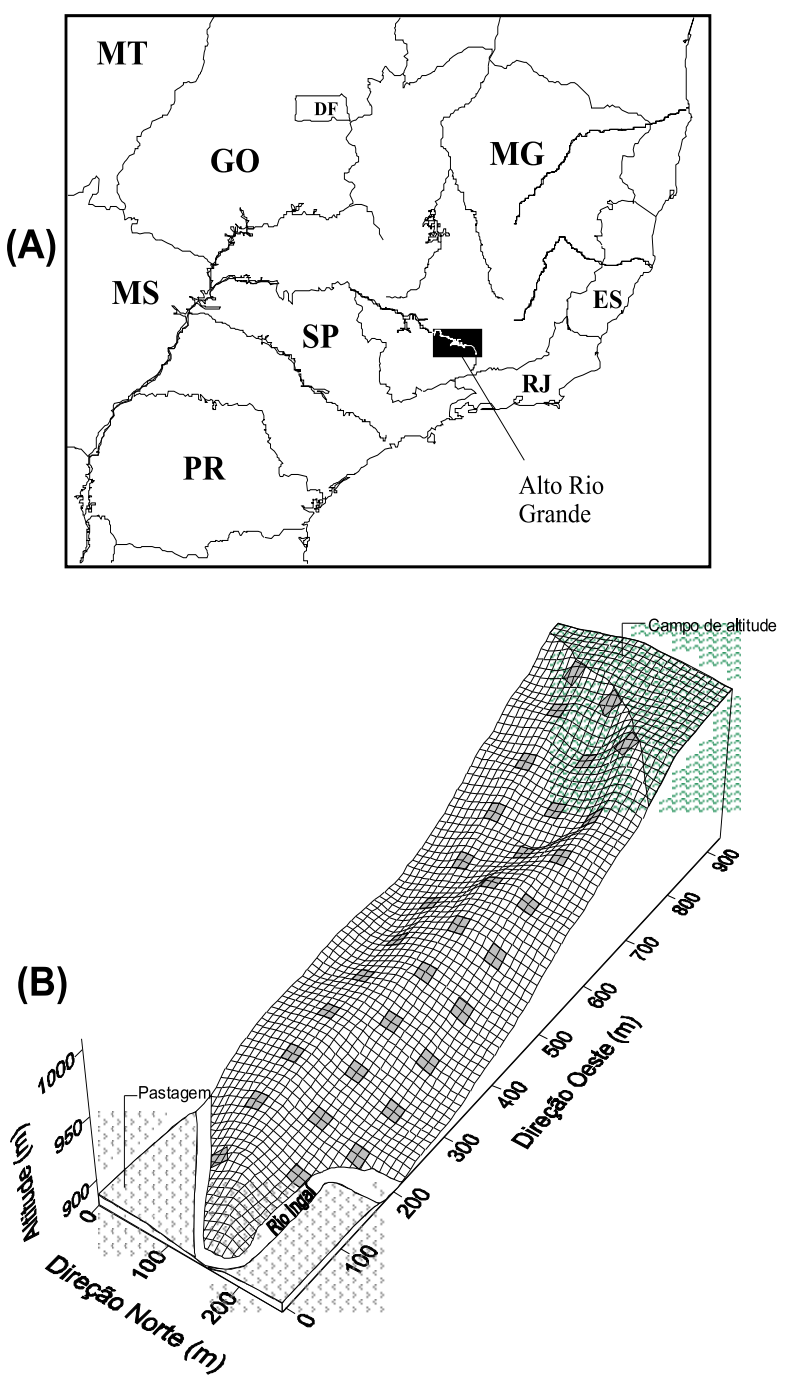

Figura 1 - (A) Situação geográfica da Mata do Galego, Município de Luminárias, na região do Alto Rio Grande, MG; e (B) mapa topográfico mostrando a distribuição das 32 parcelas de $20 \times 20 \mathrm{~m}$. Os solos das seis parcelas próximas ao rio são Argissolo Vermelho fase média fertilidade, os das 20 parcelas da meia encosta são de Argissolo Vermelho fase baixa fertilidade e os das seis da alta encosta são de Cambissolo Háplico.

Figure 1 - (A) Geographic situation of the Mata do Galego, municipality of Luminárias, Alto Rio Grande Region, Minas Gerais; SE Brazil, and (B) topographic map showing the distribution of the $3220 \times 20$ m sample plots. The soils of the six plots near the river are mesotrophic Red Argisols, those of the 20 mid slope plots are dystrophic Red Argisols, and those of the six upper slope plots are dystrophic Haplic Cambisols.
O clima da região é do tipo $\mathrm{Cwb}$, segundo a classificação de Köppen, com verão úmido e inverno seco. A temperatura média anual, de acordo com dados da Estação Meteorológica de Lavras, é de 19,61 ${ }^{\circ} \mathrm{C}$, com médias mensais variando entre $16,0^{\circ} \mathrm{C}$ (julho) e $21,8^{\circ} \mathrm{C}$ (janeiro). A precipitação anual média era de $1.517 \mathrm{~mm}$.

Nas parcelas próximas ao rio, a geologia dominante é representada pelo gnaisse granítico mesocrático e os solos mais típicos são os Argissolos Vermelhos; já na alta encosta predominam rochas metapelíticas pobres e os solos mais característicos são Cambissolos Háplicos; e, na meia encosta a geologia era representada por uma mistura dessas rochas e os solos dominantes eram os Argissolos Vermelhos.

\subsection{Levantamento das variáveis ambientais}

Foram coletadas amostras simples de solo superficial (0-20 cm de profundidade) no centro geométrico de 32 parcelas. As análises químicas e granulométricas foram realizadas no Laboratório de Análise de Solos da Universidade Federal de Lavras, e os solos foram classificados de acordo com o Sistema Brasileiro de Classificação de Solos. O levantamento topográfico foi realizado com o auxílio de hipsômetro de BlumeLeis e trena. Foram anotados os desníveis entre os vértices das parcelas e entre as parcelas. Com essas medidas, foram traçadas curvas de nível em intervalos de $2 \mathrm{~m}$ a partir da margem do rio e confeccionado o gráfico de superfície da área (Figura 1B). Foram calculados o desnível, a altitude média e a distância horizontal até o rio, para cada parcela. Com a multiplicação dessas três variáveis, obteve-se a variável produto “drenagem". A altitude média foi obtida pela média aritmética simples das altitudes dos vértices das parcelas. O desnível de cada parcela, representando a declividade média do terreno, foi obtido pela diferença entre os valores de altitude máximos e mínimos. As médias das variáveis pedológicas e topográficas foram comparadas pelo teste de Tukey. Para produzir uma ordenação ambiental das parcelas a partir das variáveis pedológicas e topográficas, foi realizada uma análise de componentes principais (PCA). Embora a PCA tenha limitações na ordenação de dados florísticos, por requerer variáveis de distribuição normal e devido ao "efeito de ferradura" produzido por uma relação quadrática entre o primeiro e o segundo eixo da ordenação, ela é de grande eficiência para explicar a variação de dados ambientais. Entre 
as variáveis pedológicas e topográficas disponíveis, aquelas redundantes, ou que pouco contribuíram para a variação total, foram eliminadas. Assim, a matriz analisada contém as variáveis: altitude, desnível, $\mathrm{pH}$, $\mathrm{K}, \mathrm{Ca}+\mathrm{Mg}, \mathrm{Al}, \mathrm{Ca}+\mathrm{Mg} / \mathrm{Al}$, soma de bases, saturação por alumínio, matéria orgânica e areia. As variáveis expressas em proporção foram transformadas pela expressão $\operatorname{Arcsen} \sqrt{x / 100}$. Para a construção do diagrama, foram utilizados os tipos de solos para verificar sua correspondência com os padrões emergentes da PCA.

\subsection{Correlação entre espécies e variáveis ambientais}

Para identificar padrões de distribuição das espécies que pudessem refletir variações ambientais, foram utilizados dois métodos de ordenação: análise de correspondência retificada (DCA) e análise de correspondência canônica (CCA). Na DCA foram utilizadas apenas as 43 espécies com mais de 15 indivíduos na amostra total. A seleção de espécies com maior número de indivíduos se justifica, principalmente, pelo fato de as espécies menos abundantes contribuírem pouco para a análise dos dados e aumentarem desnecessariamente o volume de cálculos. Os valores da matriz de abundância das espécies foram transformados pelo seu logarítmico natural $\left(x^{\prime}=1 n(x+1)\right)$. A transformação logarítmica dos valores é uma forma de padronizar os dados e é conveniente em dados de abundância de espécies, nos quais é comum ocorrerem poucos valores elevados e muitos valores baixos. Após a obtenção dos diagramas de ordenação, foi utilizada uma segunda matriz contendo as variáveis ambientais nominal e quantitativa, para que se pudesse comparar e correlacionar, a posteriori, com a distribuição das espécies. Na CCA, a matriz de espécies foi a mesma utilizada pela DCA. A segunda matriz de variáveis ambientais foi a mesma utilizada pela PCA, acrescida das variáveis distância e drenagem. Após realizar uma CCA preliminar, foram eliminadas as variáveis redundantes ou que apresentaram baixa correlação $(<0,5)$ com os eixos 1 e 2, sendo elas: altitude, desnível, distância, $\mathrm{K}, \mathrm{Ca}+\mathrm{Mg}, \mathrm{Ca}+\mathrm{Mg} / \mathrm{Al}$ e saturação por alumínio e matéria orgânica. Para confirmar os padrões indicados na CCA, foram calculados os coeficientes de corrrelação de Spearman $\left(r_{\mathrm{s}}\right)$ entre as 43 espécies e as cinco variáveis ambientais selecionadas na CCA final.

\section{RESULTADOS E DISCUSSÃO}

Na Mata do Galego, encontraram-se 200 espécies e 2.343 indíviduos distribuídos em 32 parcelas de 20 x 20 m (RODRIGUES et al., 2003).

\subsection{Variáveis ambientais}

Foram identificadas duas classes de solos: Cambissolo Háplico e Argissolo Vermelho (este dividido em dois grupos: fase média fertilidade, com saturação por bases $>35 \%$; e fase baixa fertilidade, com saturação por bases $\leq 35 \%$ ) (Quadro 1). As variáveis $\mathrm{pH}$, saturação por bases, saturação por alumínio e areia foram as únicas significativamente diferentes entre os três grupos de solos. As variáveis $\mathrm{Ca}, \mathrm{Mg}, \mathrm{Ca}+\mathrm{Mg}, \mathrm{K}$ e soma de bases apresentaram médias significativamente maiores no grupo Argissolo Vermelho fase média fertilidade. Nesse grupo, os teores de $\mathrm{Al}$ foram baixos, resultando em valores da razão $\mathrm{Ca}+\mathrm{Mg} / \mathrm{Al}$ significativamente maiores. A única variável que não mostrou diferença significativa entre os grupos de solos foi a matéria orgânica. As variáveis Al, saturação por alumínio e areia aumentaram ao longo do gradiente Argissolo Vermelho $\rightarrow$ Cambissolo Háplico, que coincide com o aumento da altitude, à exceção da parcela 10, que apresentou alta porcentagem de areia (58\%). No entanto, o pH, soma de bases e a relação $\mathrm{Ca}+\mathrm{Mg} / \mathrm{Al}$ diminuíram ao longo desse gradiente. Esse resultado indica que a topografia provavelmente influenciou as características químicas e físicas do solo. Os solos da parte mais íngreme da encosta são mais rasos, mais arenosos e, conseqüentemente, mais fortemente drenados, o que ocasionou maior concentração de $\mathrm{Al}$ e baixos níveis de $\mathrm{Ca}+\mathrm{Mg}$. Esse gradiente pode ser observado com clareza na ordenação das parcelas resultantes da análise de componentes principais (PCA) (Figura 2). Os autovalores dos quatro primeiros componentes da PCA foram 6,48, 2,08, 0,74 e 0,48 e a porcentagem de variância acumulada, $58,96 \%$, $77,86 \%, 84,59 \%$ e $89,00 \%$ para esses componentes, respectivamente. Verifica-se que os dois primeiros componentes explicaram a maior parte da variação dos dados ambientais nas parcelas.

A ordenação das parcelas, por vetores das variáveis ambientais, nos dois primeiros componentes da PCA, indica que areia e saturação por $\mathrm{Al}$, areia e $\mathrm{Al}$ apresentaram alta correlação positiva entre si e estão correlacionadas negativamente com as variáveis $\mathrm{pH}$ e com aquelas relacionadas à fertilidade $(\mathrm{K}, \mathrm{Ca}+\mathrm{Mg}, \mathrm{Ca}+\mathrm{Mg} / \mathrm{Al}$, soma de bases). Essas últimas também mostraram alta correlação positiva entre si. O primeiro componente teve maior 
correlação positiva com areia, saturação por alumínio e Al, e maior correlação negativa com $\mathrm{pH}, \mathrm{K}, \mathrm{Ca}+\mathrm{Mg}$, $\mathrm{Ca}+\mathrm{Mg} / \mathrm{Al}$ e soma de bases. $\mathrm{O}$ segundo componente foi negativamente correlacionado com matéria orgânica, altitude e desnível. Esses dois componentes dividiram as parcelas em três grupos, com base na fertilidade, textura dos solos e topografia. Um grupo é composto por parcelas sobre Cambissolo Háplico, mais arenoso, com alta saturação por alumínio e baixa fertilidade; nesse grupo, está a parcela 20, que se distanciou das demais devido ao seu alto valor em matéria orgânica, $8 \mathrm{dag} / \mathrm{kg}$, que corresponde ao dobro da maior média das outras parcelas. As parcelas sobre Cambissolos Háplico ocupam o terço superior da encosta, de topografia mais acentuada. Encontram-se aí as parcelas de borda (20, 09 e 32), que fazem interface com o campo de altitude. O segundo grupo é composto por parcelas sobre Argissolo Vermelho fase média fertilidade, e o terceiro grupo compreende o Argissolo Vermelho fase baixa fertilidade. As parcelas 01, 10 e 21 desse último grupo estão localizadas na margem do rio; as demais estão distribuídas no terço médio superior e terço médio inferior da encosta. A parcela 26 se apresentou isolada das demais devido ao seu alto valor de matéria orgânica. Adiscriminação dos grupos de solos das parcelas pela PCA, baseada nas variáveis ambientais, mostrou-se coerente com as diferenças indicadas entre eles, pelo teste de Tukey. Isso reforça a eficiência da classificação dos solos em identificar ambientes ecologicamente significativos.

Quadro 1 - Comparação entre médias em três grupos de solo das variáveis ambientais amostradas em 32 parcelas de 20 $\times 20$ m na Mata do Galego (Luminárias, MG). Os números são médias \pm o desvio-padrão e amplitude (entre parênteses). Valores seguidos de mesma letra nas linhas não diferem entre si pelo teste de Tukey

Table 1 - Comparison among means in three soil groups of environmental variables surveyed in 32 sample plots $(20 \times$ $20 \mathrm{~m}$ ) in the Mata dos Galegos (Luminárias, SE Brazil). Figures are means \pm standard deviations and range (within parentheses). Values in a row followed by the same lower case letter do not differ by Tukey tests

\begin{tabular}{|c|c|c|c|c|c|}
\hline Variáveis & $\begin{array}{c}\text { Argissolo Vermelho } \\
\text { fase média fertilidade } \\
\mathrm{N}=6\end{array}$ & $\begin{array}{c}\text { Grupos de Solos } \\
\text { Argissolo Vermelho } \\
\text { fase baixa fertilidade } \\
\mathrm{N}=20\end{array}$ & $\begin{array}{c}\text { Cambissolo } \\
\text { Háplico } \\
\mathrm{N}=6 \\
\end{array}$ & $\mathrm{~F}$ & $\mathrm{P}$ \\
\hline Altitude (m) & $\begin{array}{c}915,82 \pm 17,26 a \\
(890,3-935,1)\end{array}$ & $\begin{array}{c}919,97 \pm 24,91 a \\
(882,7-960,4)\end{array}$ & $\begin{array}{c}980,13 \pm 13,39 b \\
(962,4-993,6)\end{array}$ & 18,72 & 0,00 \\
\hline Desnível (m) & $\begin{array}{c}6,67 \pm 2,26 \text { a } \\
(3,3-9)\end{array}$ & $\begin{array}{c}5,47 \pm 2,08 \mathrm{a} \\
(2,5-10,4)\end{array}$ & $\begin{array}{c}11,03 \pm 3,21 b \\
(7,8-16,3)\end{array}$ & 12,99 & 0,00 \\
\hline pH em H2O & $\begin{array}{c}6,07 \pm 0,48 a \\
(5,6-6,8)\end{array}$ & $\begin{array}{c}4,95 \pm 0,31 b \\
(4,5-5,7)\end{array}$ & $\begin{array}{c}4,30 \pm 0,22 \mathrm{c} \\
(4-4,6)\end{array}$ & 44,04 & 0,00 \\
\hline$P(\mathrm{mg} / \mathrm{dm} 3)$ & $\begin{array}{l}1 \pm 0 \mathrm{a} \\
(1-1)\end{array}$ & $\begin{array}{c}1,25 \pm 0,44 \mathrm{a} \\
(1-2)\end{array}$ & $\begin{array}{c}2,5 \pm 0,84 b \\
(2-4)\end{array}$ & 17,25 & 0,00 \\
\hline $\mathrm{K}\left(\mathrm{mg} / \mathrm{dm}^{3}\right)$ & $\begin{array}{c}187,30 \pm 55,80 a \\
(106-243)\end{array}$ & $\begin{array}{c}57 \pm 24,63 b \\
(27-123)\end{array}$ & $\begin{array}{c}49,17 \pm 9,41 b \\
(36-64)\end{array}$ & 45,0 & 0,00 \\
\hline $\mathrm{Ca}\left(\mathrm{cmolc} / \mathrm{dm}^{3}\right)$ & $\begin{array}{c}3,367 \pm 0,87 a \\
(2,2-4,5)\end{array}$ & $\begin{array}{c}1,06 \pm 0,57 b \\
(0,4-2,7)\end{array}$ & $\begin{array}{c}0,5 \pm 0,14 \mathrm{~b} \\
(0,3-0,7)\end{array}$ & 44,04 & 0,00 \\
\hline $\mathrm{Mg}\left(\mathrm{cmolc} / \mathrm{dm}^{3}\right)$ & $\begin{array}{l}1,5 \pm 0,5 a \\
(0,6-2,1)\end{array}$ & $\begin{array}{c}0,635 \pm 0,49 b \\
(0,1-1,6)\end{array}$ & $\begin{array}{c}0,183 \pm 0,04 b \\
(1-2)\end{array}$ & 13,31 & 0,00 \\
\hline $\mathrm{Ca}+\mathrm{Mg}\left(\mathrm{cmolc} / \mathrm{dm}^{3}\right)$ & $\begin{array}{c}4,87 \pm 1,03 \text { a } \\
(3,5-6)\end{array}$ & $\begin{array}{c}1,70 \pm 1,03 b \\
(0,5-4,3)\end{array}$ & $\begin{array}{c}0,68 \pm 0,17 b \\
(0,4-0,9)\end{array}$ & 34,81 & 0,00 \\
\hline Al (cmolc/dm3) & $\begin{array}{c}0,08 \pm 0,04 \mathrm{a} \\
(0-0,1)\end{array}$ & $\begin{array}{l}0,86 \pm 0,39 b \\
(0,2-1,6)\end{array}$ & $\begin{array}{l}2,28 \pm 0,81 \mathrm{c} \\
\quad(1,5-3,8)\end{array}$ & 35,7 & 0,00 \\
\hline $\mathrm{Ca}+\mathrm{Mg} / \mathrm{Al}\left(\mathrm{cmolc} / \mathrm{dm}^{3}\right)$ & $\begin{array}{c}41,83 \pm 22,62 a \\
(35-60)\end{array}$ & $\begin{array}{l}3,36 \pm 3,94 b \\
(0,38-15,5)\end{array}$ & $\begin{array}{l}0,33 \pm 0,14 b \\
(0,18-0,53)\end{array}$ & 38,22 & 0,00 \\
\hline Soma de bases $\left(\mathrm{cmolc} / \mathrm{dm}^{3}\right)$ & $\begin{array}{c}5,33 \pm 0,97 a \\
(4-6,3)\end{array}$ & $\begin{array}{l}1,84 \pm 1,06 b \\
(0,6-4,5)\end{array}$ & $\begin{array}{c}0,8 \pm 0,18 b \\
(0,5-1)\end{array}$ & 40,67 & 0,00 \\
\hline Saturação por bases (\%) & $\begin{array}{c}48,05 \pm 7,10 a \\
(36,6-55,8)\end{array}$ & $\begin{array}{c}17,04 \pm 9,11 b \\
(4,9-34,5)\end{array}$ & $\begin{array}{c}5,72 \pm 1,33 \mathrm{c} \\
(4-7,5)\end{array}$ & 41,07 & 0,00 \\
\hline Saturação por alumínio (\%) & $\begin{array}{c}1,55 \pm 0,82 a \\
(0-2,4)\end{array}$ & $\begin{array}{c}35,94 \pm 20,42 b \\
(5,7-70,3)\end{array}$ & $\begin{array}{l}72,77 \pm 7,76 \mathrm{c} \\
(63,1-81,5)\end{array}$ & 34,16 & 0,00 \\
\hline Matéria orgânica (dag/kg) & $\begin{array}{c}3,48 \pm 0,93 \\
(1,9-4,3)\end{array}$ & $\begin{array}{c}3,13 \pm 0,63 \\
(2,1-4,8)\end{array}$ & $\begin{array}{c}4,10 \pm 2,00 \\
(2,6-8)\end{array}$ & 2,03 & 0,15 \\
\hline Areia (\%) & $\begin{array}{c}22,17 \pm 7,19 a \\
(16-36)\end{array}$ & $\begin{array}{c}32,20 \pm 10,00 \mathrm{~b} \\
(21-58)\end{array}$ & $\begin{array}{c}49,67 \pm 6,19 c \\
(42-55)\end{array}$ & 15,12 & 0,00 \\
\hline Argila (\%) & $\begin{array}{l}31,1 \pm 9,55 \text { a } \\
(18-44)\end{array}$ & $\begin{array}{c}32,8 \pm 6,26 \text { a } \\
(20-43)\end{array}$ & $\begin{array}{c}18,5 \pm 3,7 b \\
(16-24)\end{array}$ & 14,40 & 0,00 \\
\hline Silte $(\%)$ & $\begin{array}{c}39,4 \pm 7,94 \text { a } \\
(23-49)\end{array}$ & $\begin{array}{c}35,1 \pm 6,88 b \\
(22-46)\end{array}$ & $\begin{array}{c}32,8 \pm 5,5 b \\
(28-40)\end{array}$ & 14,40 & 0,01 \\
\hline
\end{tabular}




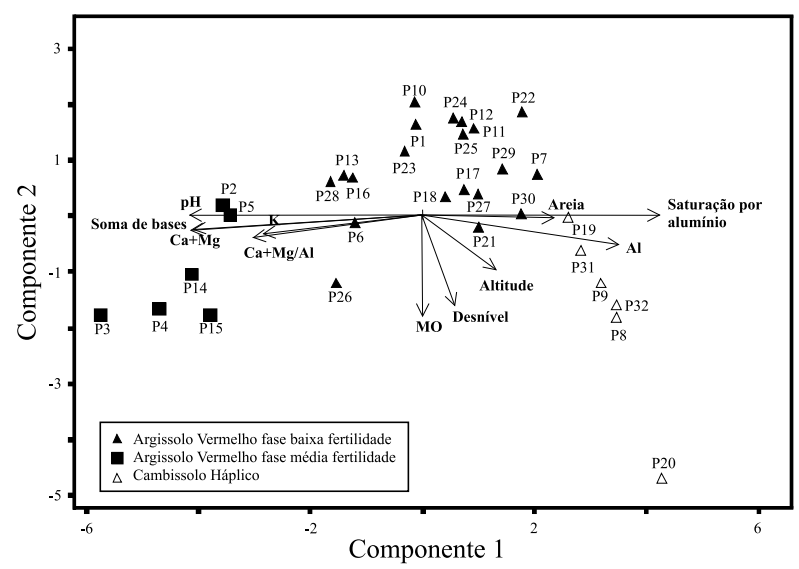

Figura 2 - Diagrama de ordenação produzido pela análise de componentes principais (PCA) mostrando a distribuição das 32 parcelas usadas para amostrar a Mata do Galego, Luminárias, MG nos dois primeiros eixos de ordenação, de acordo com suas variáveis topográficas e edáficas. Os grupos de solos das parcelas são evidenciados para facilitar a interpretação.

Figure 2 - Ordination diagram yielded by principal components analysis (PCA) showing the distribution of the 32 sample plots used to survey the Mata dos Galegos, Luminárias, SE Brazil, in the first two ordination axes, according their topographic and soil variables. Soil groups are set off to help the interpretation.

\subsection{Distribuição das espécies e variáveis ambientais}

Os autovalores dos três eixos da CCA foram baixos, e a variância total explicada foi de apenas 28,8\% (Quadro 2), indicando que as variáveis florísticas utilizadas contêm muito ruído ("noise”), ou seja, alta proporção de variância não explicada, o que é muito comum em dados de vegetação (TER BRAAK, 1987). No entanto, isso não compromete os resultados, já que as correlações ente a abundância das espécies e as variáveis ambientais foram altas e significativamente correlacionadas para os dois primeiros eixos, pelo teste de permutação de Monte Carlo. No Quadro 3, apresentam-se as correlações entre os dois primeiros eixos da CCA e as variáveis ambientais, e destas entre si. O primeiro eixo foi positivamente correlacionado com $\mathrm{pH}$ e soma de bases e negativamente com a drenagem, $\mathrm{Al}$ e areia. $\mathrm{O}$ segundo eixo foi mais fortemente correlacionado com areia e drenagem, mas as correlações foram inferiores às do primeiro eixo. As correlações entre as variáveis em geral foram altas $(>0,4)$, exceto entre drenagem e soma de bases.

R. Árvore, Viçosa-MG, v.31, n.1, p.25-35, 2007
Quadro 2 - Resumo da análise de correspondência canônica (CCA) de 32 parcelas de 20 x $20 \mathrm{~m}$ usadas para amostrar a Mata do Galego em Luminárias, MG. Valores de correlação espécie-ambiente em negrito são significativos pelo teste de permutação de Monte Carlo $(\mathrm{P}<0,05)$

Table 2 - Summary of canonical correspondence analysis (CCA) for 32 plots $(20 \times 20 \mathrm{~m})$ used to survey the Mata dos Galegos, Luminárias, SE Brazil. Speciesenvironment correlations given in bold are significant by Monte Carlo permutation tests $(P<0.05)$

\begin{tabular}{lcccc}
\hline & Eixo & Eixo & Eixo & Variância \\
& 1 & 2 & 3 & total \\
\hline Autovalores & 0,358 & 0,094 & 0,063 & 1,791 \\
$\begin{array}{l}\text { Variância acumulada } \\
\text { para dados de espécies (\%) }\end{array}$ & 20,0 & 25,3 & 28,8 & \\
$\begin{array}{l}\text { Correlação } \\
\text { espécie-ambiente }\end{array}$ & $\mathbf{0 , 9 3 0}$ & $\mathbf{0 , 8 4 3}$ & 0,795 & \\
$\begin{array}{l}\text { Teste de permutação } \\
\text { de Monte Carlo P }\end{array}$ & 0,010 & 0,030 & 0,070 & \\
\hline
\end{tabular}

O padrão de distribuição das espécies e parcelas foi semelhante nos diagramas da CCA e DCA (Figuras 3 e 4). Contudo, houve inversão no sentido do gradiente: as parcelas e espécies do lado direito dos diagramas da DCA aparecem do lado esquerdo dos diagramas da CCA e com uma disposição mais agrupada. Provavelmente, essa distribuição mais agrupada das parcelas e espécies no diagrama da CCA seja devida às diferenças entre os dois métodos de ordenação nos cálculos da extração dos eixos, sendo esta também influenciada pelas variáveis ambientais na CCA. Em ambos os métodos de ordenação, o mesmo gradiente identificado pela PCA ficou evidenciado, ou seja, os três grupos de solos se sucedem no espaço de ordenação: Argissolo Vermelho fase média fertilidade $\rightarrow$ Argissolo Vermelho fase baixa fertilidade $\rightarrow$ Cambissolo Háplico. Assim como na PCA, na CCA e na DCA as parcelas localizadas em áreas de transição com o campo de altitude foram as que mais se diferenciaram das demais. Dalanesi et al. (2004) encontraram resultado semelhante na Reserva Biológica de Poço Bonito (Lavras, MG) e, segundo esses autores, a menor profundidade do solo parece ser o principal fator determinando a transição para o campo rupestre. Investigando as propriedades químicas e físicas de solos de mata e campo, na região do Alto Rio Grande. Spera et al. (1996) observaram que a transição abrupta entre essas duas formações vegetais reflete a mudança imediata do tipo de solo, que nos locais estudados passa de Cambissolo para Latossolo ou 
Argissolo em menos de $5 \mathrm{~m}$. Segundo esses autores, características como menor profundidade do solo e menor condutividade hidráulica saturada, condicionada por maior densidade do solo, menor porosidade total e macroporosidade, restringem o avanço da mata para as áreas de campo.

Nos diagramas da CCA e DCA, as espécies Lithraea molleoides, Guazuma ulmifolia, Chrysophyllum marginatum, Machaerium hirtum, Rollinia dolabripetala e Albizia polycephala se encontram em um grupo relacionado às parcelas sobre Argissolo Vermelho fase média fertilidade, indicando uma correlação positiva com soma de bases e $\mathrm{pH}$. Algumas dessas espécies têm sido consideradas como indicadoras de solos férteis ou ocorrem preferencialmente em solo com fertilidade média a alta, com L. molleoides, $G$. ulmifolia e M. minutiflorum (RATTER et al., 1978). No lado oposto dos diagramas, estão as espécies Clethra scabra, Faramea cyanea, Sclerolobium rugosume Tapirira obtusa, relacionadas às parcelas sobre Cambissolo Háplico, indicando alta correlação com drenagem, areia e Al. A espécie C. scabra é considerada de distribuição restrita, mas com alta densidade, indicando que ela pode estar adaptada a condições restritas de "habitat" (DURIGAN et al., 2000); na Mata do Galego foram amostrados 25 indivíduos, sendo que todos ocorreram nas três parcelas da borda, que fazem interface com campo de altitude, indicando uma adaptação dessa espécie a esse tipo de ambiente. Informações da literatura reforçam essa tendência, como a observação de Pagano e Durigan (2000), que citaram essa espécie como uma das mais importantes em uma área de estudo, cujo solo é ácido, com baixos teores de Ca, Mg e K e alta saturação por alumínio, características semelhantes àquelas apresentadas pela área de ocorrência dessa espécie na Mata do Galego. Assim como C. scabra, $S$. rugosum foi amostrada apenas nas parcelas sobre Cambissolo Háplico. Já as espécies $F$. cyanea e T. obtusa ocorreram também em parcelas sobre Argissolo Vermelho, porém em baixa densidade. Esses padrões de distribuição indicados pela CCA e DCA são coerentes com os coeficientes de correlação de Spearman (Quadro 4). As espécies C. scabra, S. rugosum, F. cyanea e T. obtusa foram as mais significativas e positivanente correlacionadas com a drenagem. Já as espécies $L$. molleoides, G. ulmifolia, M. minutiflorum e M. hirtum ficaram no outro extremo da tabela, com uma correlação significativa e negativa com essa variável.

Entre as 43 espécies, 14 não apresentaram correlação significativa com nenhuma das variáveis e 8 apresentaram correlação com todas elas. As variáveis $\mathrm{pH}$ e areia exibiram os maiores números de correlações significativas, 20 e 18, respectivamente. A variável Al teve o terceiro maior número de correlações significativas (16), seguida por soma de bases (15) e drenagem (14). Entre as espécies que não apresentaram correlação com nenhuma variável, Rollinia sylvatica aparece nos diagramas da CCA e DCA claramente relacionada à parcela 15 , sendo que, no diagrama da DCA, ela aparece isolada das demais espécies. Na Figura 4, observa-se que, entre as parcelas de Argissolo Vermelho fase média fertilidade, a parcela 15 foi a única que apresentou uma maior correlação com areia. Isso se deve ao fato dessa parcela estar localizada no terço médio superior da encosta, onde Argissolo Vermelho ocorre em fase rasa, cascalhenta, com maior proporção de silte e areia. Entre os 24 indivíduos de $R$. sylvatica, amostrados 17 ocorreram em duas parcelas nessas condições.

Quadro 3 - Correlações "intraset" das variáveis ambientais com os dois primeiros eixos da CCA, e matriz de correlações ponderadas entre as variáveis. Correlações $>0,4$ são salientadas em negrito

Table 3 - Intraset correlations of the environmental variables with the first two CCA ordination axes, and matrix of weighted correlations among variables. Correlations $>0.4$ are highlighted in bold

\begin{tabular}{|c|c|c|c|c|c|c|}
\hline & \multicolumn{2}{|c|}{ Correlação "intraset" } & \multirow[b]{2}{*}{ Drenagem } & \multirow[b]{2}{*}{$\mathrm{pH}$} & \multirow[b]{2}{*}{$\mathrm{Al}$} & \multirow[b]{2}{*}{ Soma de Bases } \\
\hline & Eixo 1 & Eixo 2 & & & & \\
\hline Drenagem & $-0,871$ & $-0,464$ & & & & \\
\hline $\mathrm{PH}$ & $\mathbf{0 , 7 8 9}$ & $-0,390$ & $-0,499$ & & & \\
\hline Al & $-0,904$ & $-0,023$ & $\mathbf{0 , 7 5 5}$ & $-0,792$ & & \\
\hline Soma de Bases & 0,661 & $-0,399$ & $-0,321$ & $\mathbf{0 , 8 7 9}$ & $-0,698$ & \\
\hline Areia & $-\mathbf{0 , 8 0 0}$ & 0,478 & 0,494 & $-0,661$ & 0,593 & $-0,575$ \\
\hline
\end{tabular}


(A)

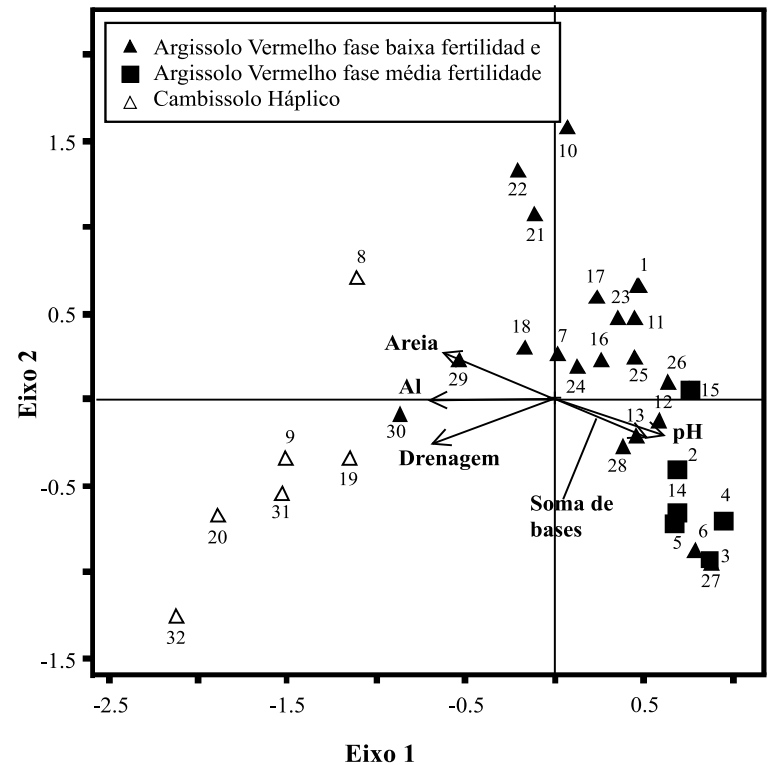

(B)

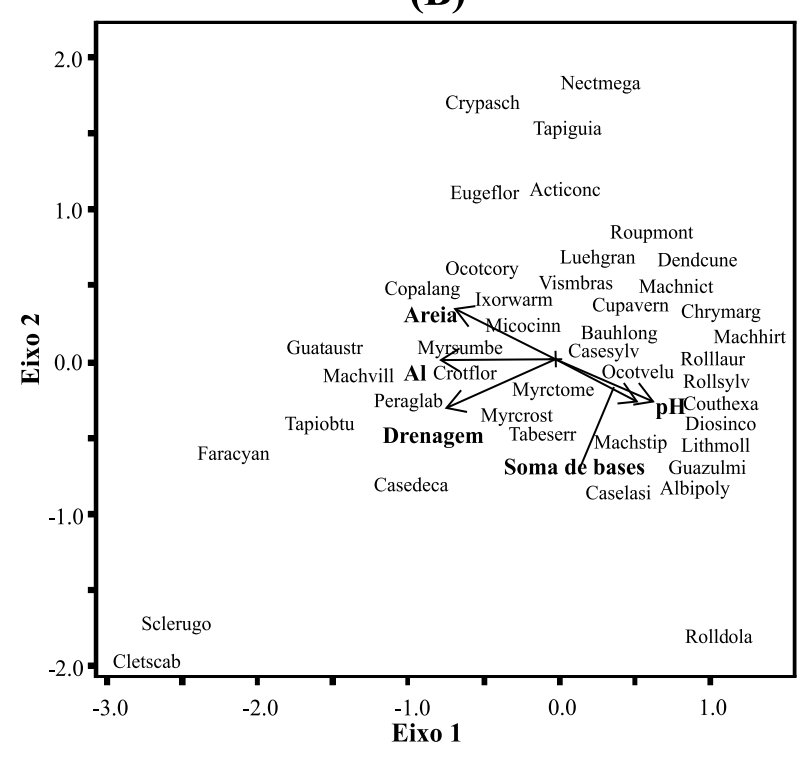

Figura 3 - Diagrama de ordenação produzido pela análise de correspondência canônica (CCA) mostrando a distribuição nos dois primeiros eixos das 32 parcelas (A) e das espécies (B), amostradas na Mata do Galego, em Luminárias, MG, correlacionadas com variáveis ambientais (vetores).

Figure 3 - Ordination diagram yielded by canonical correspondence analysis (CCA) showing the distribution on the first two axes of the 32 sample plots (A) and species surveyed in the Mata dos Galegos, Luminárias, SE Brazil, and their correlation to environmental variables (arrows).

(A)

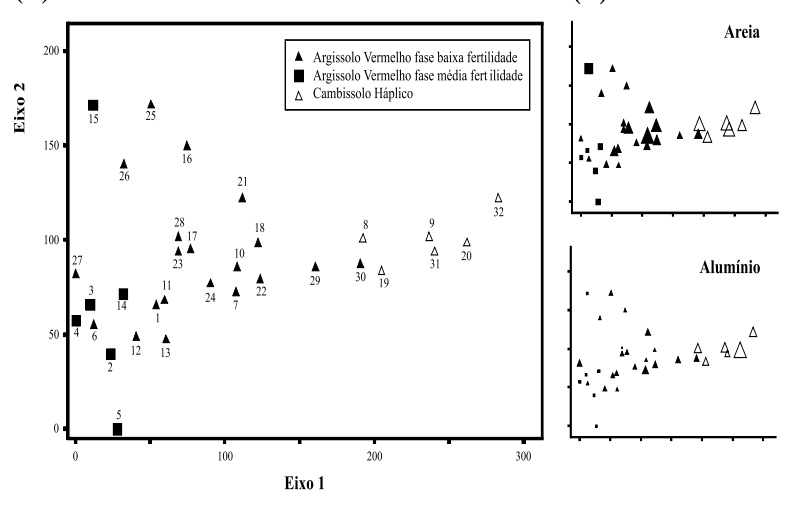

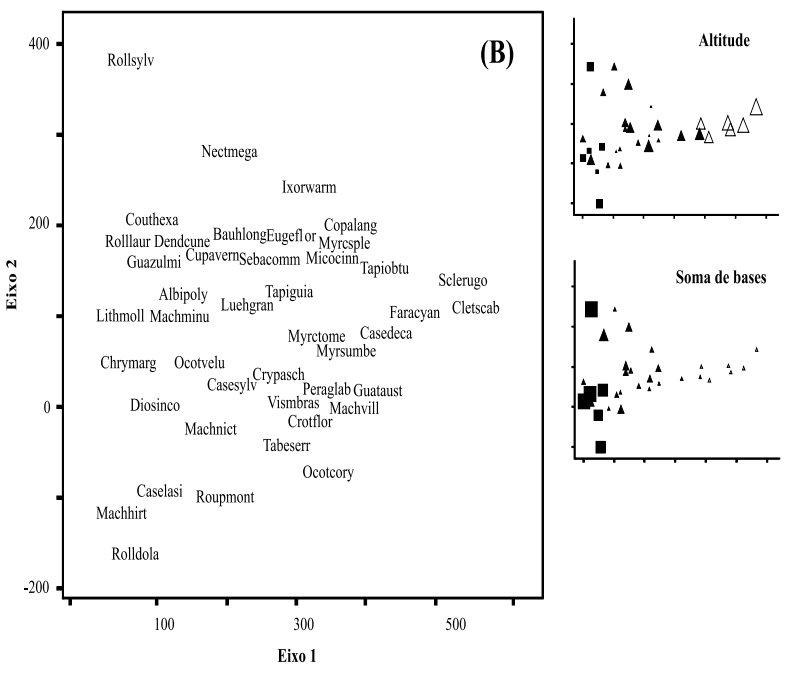

Figura 4 - Diagramas de ordenação de 32 parcelas e 43 espécies amostradas na Mata do Galego, Luminárias, MG, produzidos por análise de correspondência retificada (DCA) dos valores de abundância das espécies: (A) ordenação das parcelas, (B) espécies e (C) quatro variáveis ambientais representadas pelo tamanho dos símbolos em diagramas de ordenação das parcelas.

Figure 4 - Ordination diagrams of the 32 sample plots and 43 species surveyed in the Mata dos Galegos, Luminárias, SE Brazil, yielded by detrended correspondence analysis (DCA) of species abundance values: (A) plot ordination, $(B)$ species ordination, and $(C)$ four environmental variables plotted as symbol sizes on plot ordination diagrams.

R. Árvore, Viçosa-MG, v.31, n.1, p.25-35, 2007 
Quadro 4 - Coeficientes de correlação de Spearman $\left(r_{c}\right)$ e sua significância (entre parênteses) entre as abundâncias das 43 espécies mais abundantes e as cinco variáveis ambientais usadas na CCA. $\mathrm{N}=32$ parcelas de $20 \times 20 \mathrm{~m}$ da Mata do Galego, em Luminárias, MG. As espécies estão ordenadas por seu $\mathrm{r}_{\mathrm{s}}$ para drenagem. As correlações em negrito são significativas a $5 \%$ de probabilidade

Table 4 - Spearman correlation coefficients $\left(r_{s}\right)$ and their significance (within parentheses) between the abundances of the 43 most abundant species and the five environmental variables used in CCA. $N=32$ plots $(20 \times 20 \mathrm{~m})$ used to survey the Mata dos Galegos, Luminárias, SE Brazil. Species are ordered in descending $r_{s}$ values for drainage. Correlations in bold are significant at the level of 0.05

\begin{tabular}{|c|c|c|c|c|c|}
\hline Espécies & Drenagem & $\mathrm{pH}$ & SB & $\mathrm{Al}$ & Areia \\
\hline Faramea cyanea & $\mathbf{0 , 6 6}(0,000)$ & $\mathbf{- 0 , 7 5}(0,000)$ & $\mathbf{- 0 , 6 6}(0,000)$ & $\mathbf{0 , 7 9}(0,000)$ & $\mathbf{0 , 6 0}(0,000)$ \\
\hline Sclerolobium rugosum & $\mathbf{0 , 5 5}(0,001)$ & $\mathbf{- 0 , 5 2}(0,002)$ & $\mathbf{- 0 , 4 2}(0,016)$ & $\mathbf{0 , 5 1}(0,003)$ & $\mathbf{0 , 4 0}(0,021)$ \\
\hline Tapirira obtusa & $\mathbf{0 , 5 2}(0,003)$ & $\mathbf{- 0 , 5 9}(0,000)$ & $\mathbf{- 0 , 4 6}(0,008)$ & $\mathbf{0 , 5 9}(0,000)$ & $\mathbf{0 , 4 8}(0,005)$ \\
\hline Casearia decandra & $\mathbf{0 , 4 9}(0,004)$ & $-0,31(0,084)$ & $-0,25(0,169)$ & $0,30(0,091)$ & $0,24(0,178)$ \\
\hline Clethra scabra & $\mathbf{0 , 4 6}(0,008)$ & $\mathbf{- 0 , 4 0}(0,021)$ & $\mathbf{- 0 , 4 3}(0,014)$ & $\mathbf{0 , 4 9}(0,004)$ & $\mathbf{0 , 3 8}(0,034)$ \\
\hline Pera glabrata & $0,34(0,060)$ & $\mathbf{- 0 , 4 2}(0,015)$ & $-0,21 \quad(0,258)$ & $0,27(0,135)$ & $0,30(0,090)$ \\
\hline Croton floribundus & $0,32(0,072)$ & $-0,28(0,120)$ & $-0,26(0,143)$ & $0,22(0,225)$ & $0,19(0,288)$ \\
\hline Machaerium villosum & $0,31(0,085)$ & $\mathbf{- 0 , 5 1}(0,003)$ & $\mathbf{- 0 , 5 1}(0,003)$ & $\mathbf{0 , 5 5}(0,001)$ & $\mathbf{0 , 3 6}(0,044)$ \\
\hline Copaifera langsdorffii & $0,30(0,097)$ & $\mathbf{- 0 , 5 1}(0,003)$ & $\mathbf{- 0 , 3 1}(0,081)$ & $\mathbf{0 , 4 0}(0,022)$ & $\mathbf{0 , 6 0}(0,000)$ \\
\hline Myrcia splendens & $0,28(0,122)$ & $-0,25(0,171)$ & $-0,16(0,388)$ & $0,29(0,112)$ & $0,23(0,209)$ \\
\hline Myrsine umbellata & $0,27(0,130)$ & $\mathbf{- 0 , 3 9}(0,028)$ & $-0,33(0,061)$ & $\mathbf{0 , 3 7}(0,037)$ & $\mathbf{0 , 3 9}(0,028)$ \\
\hline Guatteria australis & $0,16(0,381)$ & $\mathbf{- 0 , 4 5}(0,010)$ & $\mathbf{- 0 , 3 6}(0,040)$ & $\mathbf{0 , 5 1}(0,003)$ & $0,32(0,074)$ \\
\hline Ocotea corymbosa & $0,16(0,392)$ & $\mathbf{- 0 , 3 8}(0,034)$ & $-0,32(0,079)$ & $0,29(0,107)$ & $0,28(0,117)$ \\
\hline Ixora warmingii & $0,14(0,427)$ & $-0,26(0,158)$ & $-0,24(0,177)$ & $0,30(0,089)$ & $0,17(0,354)$ \\
\hline Myrcia tomentosa & $0,12(0,494)$ & $0,037(0,842)$ & $-0,12(0,515)$ & $0,08(0,664)$ & $-0,03(0,890)$ \\
\hline Miconia cinnamomifolia & $0,09(0,638)$ & $-0,27(0,129)$ & $-0,27(0,138)$ & $0,207(0,256)$ & $0,200(0,273)$ \\
\hline Tabebuia serratifolia & $0,01(0,941)$ & $-0,014(0,941)$ & $-0,211(0,247)$ & $0,17(0,354)$ & $0,11 \quad(0,539)$ \\
\hline Vismia brasiliensis & $-0,01 \quad(0,993)$ & $-0,18(0,319)$ & $-0,22(0,232)$ & $0,13(0,467)$ & $0,16(0,383)$ \\
\hline Rollinia sylvatica & $-0,01 \quad(0,989)$ & $0,25(0,169)$ & $0,26(0,145)$ & $-0,27(0,132)$ & $-0,16(0,376)$ \\
\hline Albizia polycephala & $-0,02(0,906)$ & $0,48(0,006)$ & $0,48(0,006)$ & $-0,48(0,006)$ & $-0,44(0,011)$ \\
\hline Eugenia florida & $-0,05(0,785)$ & $-0,12(0,523)$ & $-0,12(0,511)$ & $0,14(0,454)$ & $0,32(0,071)$ \\
\hline Bauhinia longifolia & $-0,05(0,775)$ & $-0,03(0,882)$ & $0,05(0,782)$ & $-0,03(0,857)$ & $-0,09(0,614)$ \\
\hline Cryptocarya aschersoniana & $-0,06(0,726)$ & $-0,42(0,017)$ & $-0,39(0,027)$ & $0,35(0,053)$ & $0,32(0,076)$ \\
\hline Casearia lasiophylla & $-0,10(0,570)$ & $\mathbf{0 , 4 4}(0,012)$ & $0,24(0,182)$ & $-0,34(0,055)$ & $-0,21 \quad(0,247)$ \\
\hline Luehea grandiflora & $-0,12(0,515)$ & $-0,14(0,460)$ & $-0,16(0,384)$ & $0,11(0,564)$ & $0,04(0,815)$ \\
\hline Rollinia dolabripetala & $-0,13(0,482)$ & $\mathbf{0 , 4 5}(0,009)$ & $\mathbf{0 , 4 1}(0,019)$ & $\mathbf{- 0 , 4 0}(0,024)$ & $\mathbf{- 0 , 6 7}(0,000)$ \\
\hline Nectandra megapotamica & $-0,14(0,435)$ & $0,09(0,630)$ & $0,14(0,45$ & $-0,10(0,592)$ & $\mathbf{0 , 3 6}(0,042)$ \\
\hline Sebastiania commersoniana & $-0,17(0,348)$ & $-0,28(0,115)$ & $-0,24(0,190)$ & $0,27(0,132)$ & $0,23(0,211)$ \\
\hline Coutarea hexandra & $-0,20(0,262)$ & $0,16(0,368)$ & $0,26(0,148)$ & $-0,24(0,190)$ & $\mathbf{- 0 , 4 0}(0,022)$ \\
\hline Ocotea velutina & $-0,22(0,221)$ & $0,27(0,140)$ & $0,23(0,201)$ & $-0,28(0,124)$ & $\mathbf{- 0 , 3 7}(0,035)$ \\
\hline Guazuma ulmifolia & $-0,26(0,152)$ & $\mathbf{0 , 6 9}(0,000)$ & $\mathbf{0 , 6 4}(0,000)$ & $\mathbf{- 0 , 6 8}(0,000)$ & $\mathbf{- 0 , 6 2}(0,000)$ \\
\hline Cupania vernalis & $-0,28(0,118)$ & $0,17(0,360)$ & $0,13(0,481)$ & $-0,26(0,148)$ & $-0,12(0,516)$ \\
\hline Rollinia laurifolia & $-0,33(0,067)$ & $0,18(0,316)$ & $0,18(0,316)$ & $-0,17(0,356)$ & $\mathbf{- 0 , 4 2}(0,016)$ \\
\hline Machaerium nictitans & $-0,35(0,052)$ & $0,21 \quad(0,245)$ & $0,21 \quad(0,241)$ & $-0,22(0,219)$ & $-0,15(0,405)$ \\
\hline Machaerium minutiflorum & $\mathbf{- 0 , 3 7}(0,037)$ & $\mathbf{0 , 7 6}(0,000)$ & $\mathbf{0 , 7 0}(0,000)$ & $\mathbf{- 0 , 7 1}(0,000)$ & $\mathbf{- 0 , 6 8}(0,000)$ \\
\hline Diospyros inconstans & $\mathbf{- 0 , 3 7}(0,036)$ & $\mathbf{0 , 6 0}(0,000)$ & $\mathbf{0 , 4 9}(0,004)$ & $\mathbf{- 0 , 5 4}(0,001)$ & $\mathbf{- 0 , 6 6}(0,000)$ \\
\hline Dendropanax cuneatus & $\mathbf{- 0 , 4 1}(0,020)$ & $0,12(0,502)$ & $0,12(0,494)$ & $-0,09(0,615)$ & $-0,19(0,308)$ \\
\hline Machaerium hirtum & $\mathbf{- 0 , 4 2}(0,016)$ & $\mathbf{0 , 4 1}(0,021)$ & $\mathbf{0 , 3 8}(0,030)$ & $\mathbf{- 0 , 4 1}(0,019)$ & $-0,34(0,053)$ \\
\hline Lithraea molleoides & $\mathbf{- 0 , 4 3}(0,014)$ & $\mathbf{0 , 6 3}(0,000)$ & $\mathbf{0 , 5 8}(0,000)$ & $\mathbf{- 0 , 5 8}(0,000)$ & $\mathbf{- 0 , 6 6}(0,000)$ \\
\hline Tapirira guianensis & $\mathbf{- 0 , 4 6}(0,008)$ & $-0,14(0,440)$ & $-0,19(0,309)$ & $0,13(0,459)$ & $0,19(0,307)$ \\
\hline Casearia sylvestris & $\mathbf{- 0 , 4 7}(0,006)$ & $0,30(0,097)$ & $0,15(0,422)$ & $-0,25(0,167)$ & $-0,15(0,410)$ \\
\hline Chrysophyllum marginatum & $\mathbf{- 0 , 5 6}(0,001)$ & $\mathbf{0 , 4 4}(0,012)$ & $\mathbf{0 , 4 3}(0,014)$ & $\mathbf{- 0 , 4 5}(0,009)$ & $\mathbf{- 0 , 3 5}(0,050)$ \\
\hline Roupala brasiliensis & $\mathbf{- 0 , 6 2}(0,000)$ & $0,17(0,354)$ & $0,03(0,862)$ & $-0,14(0,457)$ & $0,04(0,825)$ \\
\hline
\end{tabular}


A maior ou menor correlação com as variáveis, em geral, refletiu-se na distribuição das espécies em função da fertilidade e textura do solo, ao longo de um gradiente topográfico. A topografia está freqüentemente relacionada às variações físicas e químicas do solo que, muitas vezes, são refletidas pela vegetação, como foi observado por Botrel et al. (2002) em uma floresta semidecidual nas margens do rio Ingaí, onde a composição florística foi significativamente correlacionada com a topografia. Em outro estudo realizado em uma floresta ripária às margens do rio Grande, em Bom Sucesso,M), a densidade das espécies também apresentou correlação significativa com a topografia e com as propriedades químicas do solo (OLIVEIRA FILHO et al., 1994b). Algumas tendências observadas por esses autores foram semelhantes às encontradas na Mata do Galego: Copaifera langsdorfii, Ixora warmingii, Tapirira obtusa e Myrsine umbellata ocorreram em solos de baixa fertilidade e Machaerium nictitans e M. minutiflorum em solos mais férteis. Por outro lado, M. villosum que, em Bom Sucesso ocorreu em solo fértil e em locais mais baixos do gradiente topográfico, na Mata do Galego foi abundante nas parcelas de maior altitude e de solos menos férteis. No entanto, essa diferença pode ser devida, em parte, ao histórico de exploração de madeira na Mata do Galego. Segundo informações locais, $M$. villosum foi uma das espécies exploradas ali,e os indivíduos amostrados no levantamento estrutural estavam todos localizados em locais de declive mais acentuado, o que pode ter auxiliado a preservação desses espécimes. O tipo de distúrbio antrópico, período de tempo em que ocorreu e a história da regeneração após o distúrbio provavelmente influenciaram a composição estrutural e florística, como observaram Metzger et al., (1997) em fragmentos de florestas ripárias ao longo do rio Jacaré-Pepira, Estado de São Paulo.

Cada espécie tem um intervalo de tolerância em relação às variáveis ambientais, e quase sempre os limites dessa tolerância não são bruscos em um gradiente ambiental. Existe um ponto ótimo a partir do qual a abundância da espécie vai diminuindo em direção aos extremos desse gradiente, que pode ser um recurso (luz, nutrientes) ou condições de "habitat" ( $\mathrm{pH}$, altitude, topografia). Provavelmente, as espécies que distanciaram dos centros dos diagramas de ordenação tenham menor tolerância em relação a algumas variáveis ambientais utilizadas e, por isso, apresentaram um padrão de distribuição mais evidente. No entanto, para caracterizar as espécies em relação ao seu "habitat" preferencial, é necessário que as tendências apresentadas pelas espécies em um estudo sejam observadas em outros locais (SOUZA et al., 2003; DALANESI et al., 2004; ROCHA et al., 2005).

\section{CONCLUSÕES}

O solo da floresta apresentou variações na textura e $\mathrm{n}$ fertilidade que, em parte, foram refletidas pela abundância de algumas espécies.

Os métodos de análise de gradientes utilizados evidenciaaram uma coerência nos padrões de distribuição das espécies em relação às variáveis ambientais.

Em geral, a distribuição da maioria das espécies apresentou correlação com fertilidade e textura do solo, ao longo de um gradiente topográfico.

\section{AGRADECIMENTOS}

Este trabalho integra o Subprojeto Estratégias para a Consevação e Manejo da Biodiversidade em Fragmentos de Florestas Semidecíduas executado pela EMBRAPA Recursos Genéticos, a Universidade Federal de Lavras (UFLA) e Universidade de Brasília (UnB), sendo um integrante do Projeto de Conservação e Utilização Sustentável da Diversidade Biológica Brasileira (PROBIO), supervisionado pelo Ministério do Meio Ambiente (MMA), gerido pelo Conselho Nacional de Desenvolvimento Científico e Tecnológico (CNPq) e apresentado pelo governo brasileiro ao GEF/BIRD.

\section{REFERÊNCIAS}

BOTREL, R.T. et al. Composição florística e estrutura da comunidade arbórea de um fragmento de floresta estacional semidecidual em Ingaí, MG, e a influência de variáveis ambientais na distribuição das espécies. Revista Brasileira de Botânica, v.25, p.195-213, 2002.

CARVALHO, D.A. et al. Variações florísticas e estruturais do componente arbóreo de uma floresta ombrófila alto-montana às margen do rio Grande, Bocaina de Minas, MG, Brasil. Acta Botanica Brasílica, v.19, n.1, p.91-109, 2005.

CLARK, D.B.; CLARK, D.A.; READ, J.M. Edaphic variation and the mesoscale distribution of tree species in a neotropical rain forest. Journal of Ecology, v.86, p.101-112, 1998. 
DALANESI, P.E.; OLIVEIRA-FILHO, A.T.; FONTES, M.A.L. Flora e estrutura do componente arbóreo da floresta do Parque Ecológico Quedas do Rio Bonito, Lavras - MG, e correlações entre distribuição das espécies e variáveis ambientais. Acta Botânica Brasílica, v.18, n.4, p.737-757, 2004.

DURIGAN, G.; RODRIGUES, R.R.; SCHIAVINI, I. A heterogeneidade ambiental definindo a metodologia de amostragem da floresta ciliar. In: RODRIGUES, R.R.; LEITÃO FILHO, H.F. (Ed.).

Matas ciliares: conservação e recuperação. São Paulo: EDUSP, 2000. p.159-167.

ESPÍRITO-SANTO, F.D.B. et al. Variáveis ambientais e a distribuição de espécies arbóreas em um remanescente de floresta estacional semidecídua montana mo campus da Universidade Federal de Lavras, MG. Acta Botânica Brasílica, v.16, n.3, p.331-356, 2002.

METZGER, J.P.; BERNACCI, L.C.; GOLDENBERG, $\mathrm{R}$. Pattern of tree species diversity in riparian forest fragments of different widths (SE Brazil). Plant Ecology, v.133, p.135-152, 1997.

OLIVEIRA FILHO, A.T. et al. Effects of canopy gaps, topography, and soils on the distribution of woody species in a Central Brazilian Deciduous Dry Forest. Biotropica, v.30, n.3, p.362-375, 1998.

OLIVEIRA FILHO, A.T.; SCOLFORO, J.R.; MELLO, J.M. Composição florística e estrutura comunitária de um remanescente de floresta semidecídua montana em Lavras, MG. Revista Brasileira de Botânica, v. 17, n.2, p.167-182, 1994a.

OLIVEIRA FILHO, A.T. et al. Differentiation of streamside and upland vegetation in area of montane semideciduous forest in south-eastern Brazil. Flora, v.189, p.287-305, 1994b.

OLIVEIRAFILHO, A.T. et al. Variation in tree community composition and structure with changes in soil properties within a fragmento of semideciduous forest in South-eastern Brazil. Edinburgh Journal Botany, v.58, n.1, p.139-158, 2001.
PAGANO, S.N.; DURIGAN, G. Aspectos da ciclagem de nutrientes em matas ciliares do Oeste do estado de São Paulo, Brasil. In: RODRIGUES, R.R.; LEITÃO FILHO, H.F. (Ed.). Matas

ciliares: conservação e recuperação. São Paulo: EDUSP, 2000. p.109-123.

RATTER, J.A. et al. Observations on forests of some mesotrophic soils in Central Brazil.

Revista Brasileira de Botânica, v.1, p.47-58, 1978.

ROCHA, C.T.V. et al. Comunidade arbórea de um continuum entre floresta paludosa e de encosta em Coqueiral, Minas Gerais, Brasil. Revista Brasileira de Botânica, v.28, n.2, p.203-218, 2005.

RODRIGUES, L.A. et al. Florística e estrutura da comunidade arbórea de um fragmento florestal em Luminárias, MG. Acta Botanica Brasílica, v.17, n.1, p.71-87, 2003.

SOUZA, J.S. et al. Análise das variações florísticas e estruturais da comunidade arbórea de um fragmento de floresta semidecídua às margens do rio Capivari, Lavras, MG. Revista Árvore, v.27, p.185-206, 2003.

SPERA, S.T.; FERREIRA, M.M.; CURI, N. Interrelações entre propriedades físico-hídricas de solos adjacentes de mata e campo no Alto Rio Grande (MG). Ciência e Agrotecnologia, v.20, n.2, p.178-182, 1996.

TER BRAAK, C.J.F. The analysis of vegetationenvironment relationships by canonical correspondence anlysis. Vegetatio, v.69, n.3, p.69-77, 1987.

van den BERG, E.; OLIVEIRA FILHO, A.T. Spatial partitioning among tree species within na área of tropical montage gallery forest in south-eastern Brazil. Flora, v.194, p.249-266, 1999. 
